\title{
A Review on Self Emulsifying Nanoemulsion
}

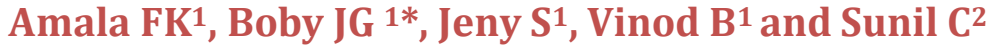 \\ 1Department of Pharmaceutics, St. Joseph's College of Pharmacy, Cherthala, Kerala, \\ India \\ 2Department of Pharmacology, St. Joseph's College of Pharmacy, Cherthala, Kerala, \\ India
}

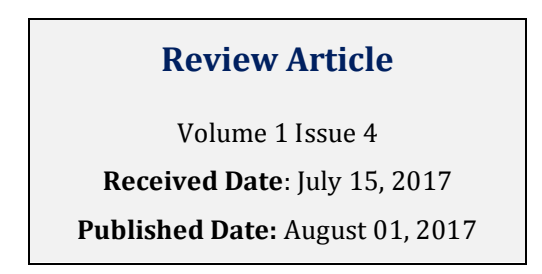

*Corresponding author: Boby Johns G, Professor, Department of Pharmaceutics, St. Joseph's College of Pharmacy, Cherthala, Kerala, India-688 524, Tel: +91 9846144409, Email: bjg712@gmail.com

\section{Abstract}

Current article is focused on recent advances in the formulation and characterization of Self Emulsifying Nanoemulsions in Drug Delivery. Self-Emulsifying Drug Delivery Systems (SEDDS) are the isotropic mixtures of oil, surfactant, co surfactant and drug that form oil in water Nanoemulsion when introduced into aqueous phase under gentle agitation. Nanoemulsions are novel drug delivery systems consisting of emulsified oil and water systems with mean droplet diameters ranging from 50 to $1000 \mathrm{~nm}$. Usually, the average droplet size is between 100 and $500 \mathrm{~nm}$ and can exist as oilin-water $(\mathrm{o} / \mathrm{w})$ or water-in-oil (w/o) form, where the core of the particle is either oil or water, respectively. A lot of techniques are available for enhancing absorption of poorly water-soluble drugs. One such approach is the use of lipidbased systems. Thus enhancement of aqueous solubility in such case is a valuable goal to successfully formulate them into bioavailable dosage forms. Nanoemulsions can be prepared by high- and low-energy methods. Both high-energy and low energy methods can produce stable Nanoemulsions. Nanoemulsion can be formulated for delivery of drugs through various routes. Nanoemulsions are well tolerated orally and on the skin and mucous membranes when used to deliver topically active drugs. Nanoemulsion globules can fuse with membranes of lipid-containing organisms facilitating penetration and transfer. Less amount of surfactant is required in Nanoemulsions compared to other emulsion systems. This can increase the bioavailability of poorly soluble drugs since small particles easily cross the absorption membrane. Furthermore, very small size provides large surface area which eases the solubilization and penetration through the skin or epithelial layer.

Keywords: Nanoemulsions; Solubilization; Nanosuspensions; Nanoparticles

\section{Introduction}

An ideal drug delivery system fulfils the objective of maximizing therapeutic effect while minimizing toxicity. With the progress in time and advances in science and technology, dosage forms have evolved from simple mixtures and pills, to highly sophisticated systems, which are known as novel drug delivery systems. 


\section{Open Access Journal of Pharmaceutical Research}

Nanoemulsions are novel drug delivery systems consisting of emulsified oil and water systems with mean droplet diameters ranging from 50 to $1000 \mathrm{~nm}$. Usually, the average droplet size is between 100 and $500 \mathrm{~nm}$ and can exist as oil-in-water (o/w) or water-in-oil (w/o) form, where the core of the particle is either oil or water, respectively. Nanoemulsions are made from pharmaceutical surfactants that are generally regarded as safe (GRAS). The surfactant type and concentration in the aqueous phase are chosen to provide good stability against coalescence. Several types of oils-natural semisynthetic and synthetic are used in the formulation of Nanoemulsions [1].

In pharmaceutical drug discovery, various new chemical entities suffer from poor water solubility. Solubility is considered one of the prerequisites for intestinal absorption; therefore, drugs with low water solubility are predisposed to low and variable oral bioavailability. Thus, an increasingly important area of pharmaceutical research is finding safe and effective methods of solubliziing Poorly Water-Soluble Drugs (PWSD). Many approaches to improving oral bioavailability have been investigated by enhancing drug solubility or increasing the surface area available for dissolution [2].

Recently, attention has been drawn toward self nanoemulsifying drug delivery systems (SNEDDS) and Solid Nanosuspensions (NS) for improving the oral bioavailability of Biopharmaceutics Classification System (BCS) class II drugs via enhancing their solubility. SNEDDS are uniform preconcentrate of oils, surfactants, and co-surfactants that form Nanoemulsion with 20-200 $\mathrm{nm}$ size range upon dilution with no need to perform a dissolution step. SNEDDS spread instantly in the gastrointestinal tract (GIT) fluids. GIT fluids and their motility provide the necessary dispersion of the Nanoemulsion [3-8]. The anhydrous nature of SNEDDS allows for the oral administration of the drug in soft or hard gelatin capsules. These nano-sized droplets offer enhancement in dissolution rates and hence bioavailability of poorly water soluble and lipophilic drugs $[9,10]$.

Nanoemulsions are optically isotropic, transparent, thermodynamically stable systems of oil, surfactants and water with miniscule droplet size, high solubilization capacity for lipophilic drugs ease of preparation, enhanced biocompatibility and size of emulsion droplets influence its target distribution thereby improve the penetration of drug into membrane. ${ }^{1}$ Nanoemulsions are formed when the interfacial tension at the oil/water interface is brought to a very low level and the interfacial layer is kept highly flexible and fluid. These two conditions are usually met by a careful and precise choice of the components and of their respective proportions and by the use of a "co-surfactant" which brings flexibility to the oil/water interface ${ }^{1}$ The capacity of Nanoemulsions to dissolve large quantities of low soluble drugs along with their mutual compatibility and ability to protect the drugs from hydrolysis and enzymatic degradation make them ideal drug delivery vectors. The major advantages of Nanoemulsions as drug delivery carriers include;

Increased drug loading,

Enhanced drug solubility and bioavailability

Reduced patient variability

Controlled drug release

Protection from enzymatic degradation [11].

A lot of techniques are available for enhancing absorption of poorly water-soluble drugs, like use of lipidbased systems. Thus enhancement of aqueous solubility in such case is a valuable goal to successfully formulate them into bioavailable dosage forms. A range of novel strategies are currently being developed for efficient delivery of poorly water-soluble drugs, such as the formulation of amorphous solid form, nanoparticles, micro emulsions, solid dispersions, melt extrusion, salt formation and formation of water-soluble complexes. Among all, the most accepted approach is the lipid-based formulation [12,13]. Lipid-based formulations enhance the absorption by enhancing solubilization, prolonging gastric residence time, stimulating the intestinal lymphatic transport pathway, altering intestinal permeability, reduced activity of efflux transporters and reduced metabolism. Lipid-based formulations present a large range of optional systems such as solutions, suspensions, self-emulsifying systems and Nanoemulsions.

Nanoemulsions can be prepared by high- and lowenergy methods. Both high-energy and low energy methods can produce stable Nanoemulsions. Highpressure homogenizer or ultra sound generator can be used for the preparation of Nanoemulsion by high-energy emulsification method. Self emulsification and phase inversion methods-phase inversion temperature and phase inversion composition are low-energy methods for the preparation of Nanoemulsions [14]. Low-energy emulsification methods depend on the phase behavior and properties of the constituents, and they utilize the stored energy of the system to form nanodroplets. The emulsification can be brought about by changing the parameters such as temperature and composition, which 


\section{Open Access Journal of Pharmaceutical Research}

would affect the hydrophilic lipophilic balance of the system [4].

\section{Nanoemulsion Based Delivery System: Types and Properties}

\section{Self Emulsifying Formulations (SEFs)}

Self-emulsifying formulations (SEFs) are mixtures of oil, surfactant, co-surfactant, and co solvents (absence of external phase water) and forms a transparent isotropic solution, which emulsify under gentle agitation similar to those which would be encountered in gastrointestinal tract (GIT). It has been recognized that this formulation when administered orally undergo spontaneous emulsification in aqueous GI fluids [15]. This emulsified oil (triglycerides) stimulates bile secretion and drug containing oil droplets are further emulsified by bile salts. Lipid droplets are then metabolized by lipases and co lipases, secreted from the salivary gland, gastric mucosa and pancreas, which also hydrolyze the triglycerides into di-and mono glycerides and free fatty acids. Further, solubilization of these molecules occurs during the passage through the GI tract and eventually forms a range of emulsion droplets; vesicular structures and mixed micelles containing bile salts, phospholipids and cholesterol [15]. Upon mixing with water the system SEFs have an ability to form fine colloidal droplets with very high surface area. In many cases, this accelerates the digestion of the lipid formulation, improves absorption, and reduces food effect and inter-subject variability [16]. Self emulsifying formulations distribute readily in the GI tract, the digestive motility of the stomach and the intestines provides sufficient agitation enough for the spontaneous formation of emulsions $[17,18]$.

SEFs prepared using surfactants of HLB $<12$ possess high stability and improved dissolution (for poorly soluble drugs) due to enhancement in surface area on dispersion. Therefore, their absorption is independent of bile secretion and ensures a rapid transport of poorly soluble drugs into the blood [15]. According to Reiss, self emulsification occurs when the entropy changes that favor dispersion is greater than the energy required to increase the surface area of the dispersion. The free energy of the conventional emulsion is a direct function of the energy required to create a new surface between the oil and water phase and can be described by the equation [19]: $\Delta 42 \mathrm{G}=\mathrm{aN}_{\mathrm{i}} \mathrm{p} \mathrm{r}_{\mathrm{i}}$. Where, $\Delta \mathrm{G}$ is the free energy associated with the process, $r_{i}$ is the radius of droplets, $N_{i}$ is the number of droplets, $p$ is the interfacial energy. The two phases of the emulsion tend to separate with time to reduce the interfacial area and thus, minimize the free energy of the system (s). The conventional emulsifying agents stabilize emulsions resulting from aqueous dilution by forming a monolayer around the emulsion droplets, reducing the interfacial energy and forming a barrier to coalescence. On the other hand, emulsification occurs spontaneously with SEDDS, as the free energy required to form the emulsion is low, whether positive or negative [20]. For emulsification to take place, it is vital for the interfacial structure to offer negligible or no resistance against surface shearing [21]. The ease of emulsification has been suggested to be related to the ease of water penetration into various liquid crystals or gel phases formed on the surface of the droplet [22-24]. The interface between the oil and aqueous continuous phases is formed upon addition of a binary mixture (oil/non-ionic surfactant) to water [22]. This is followed by solubilization within the oil phase, as a result of aqueous penetration through the interface. Invariably, this tends to occur until the solubilization limit is attained close to the inter phase. Further, aqueous penetration will lead to the formation of the dispersed liquid crystal phase. Ultimately, everything that is in close proximity with the interface will be liquid crystal, the actual amount of which depends upon the emulsifier concentration in the binary mixture. Hence, following gentle agitation of the selfemulsifying system, water rapidly penetrates into the aqueous cores leading to interface disruption and droplet formation [24].

When compared with emulsions, which are sensitive and metastable dispersed forms, SEFs are physically stable formulations that are easy to manufacture. Thus, for lipophilic drugs that exhibit dissolution rate-limited absorption, these systems may offer an improvement in the rate and extent of absorption and result in more reproducible blood time profiles [25].

SEFs have been transformed into solid dosage forms using techniques such as melt granulation, where the lipid excipients acts as a binder and solid granules are produced on cooling. Solvents or supercritical fluids can be used with semisolid excipients, which are solubilized and then the solvent evaporated to produce a waxy powder. Spraying techniques can be used to produce powder formulations. These techniques enable the production of granules or powders that can then be compressed into a tablet form or filled into capsules. In all cases, the lipid excipients used must be semi-solid at room temperature [26].

Self emulsifying drug delivery systems (SEDDS): Selfemulsifying drug delivery system (SEDDS) is a strategy that has drawn wide research interest, basically due to its distinct capacity to solubilize and improve the 


\section{Open Access Journal of Pharmaceutical Research}

bioavailability of hydrophobic drugs. This it does by ensuring aqueous solubility of the lipophilic drug [27]. The presence of oil makes SEDDS unique and distinguishes them from ordinary surfactant dispersions of drugs [28]. SEDDS are isotropic combination of drug, lipid/oil, co solvents and surfactants [27]. On dilution by an aqueous phase they form fine stable oil-in-water $(\mathrm{o} / \mathrm{w})$ emulsions or fine lipid droplets which is the characteristic feature of these systems. When such a formulation is released into the lumen of the GIT, it disperses to form a fine emulsion generally $\mathrm{o} / \mathrm{w}$ emulsion. SEDDS are generally formulated with triglyceride oils and ethoxylated nonionic surfactants. In general, the concentration of surfactant is greater than $25 \%$ in the formulation. The size of droplets ranges approximately less than $100 \mathrm{~nm}$ [27]. SEDDS are believed to be superior compared with lipid solutions due to the presence of surfactants in the formulations leading to a more uniform and reproducible bioavailability [16].

Advantages of SEDDS include:

More consistent drug absorption.

Selective targeting of drug(s) toward specific absorption window in GIT.

Protection of drug(s) from the gut environment.

Control of delivery profiles.

Reduced variability including food effects.

Enhanced oral bioavailability enabling reduction in dose and high drug loading efficiency [29].

Self emulsifying formulations spread readily in the gastrointestinal tract (GIT), and the digestive motility of the stomach and intestine provide the agitation necessary for self emulsification. These systems advantageously present the drug in dissolved form and the small droplet size provides a large interfacial area for the drug absorption. SEDDSs typically produce emulsions with turbid appearance, and droplet size between $200 \mathrm{~nm}$ to 5 $\mu \mathrm{m}$ while self micro emulsifying drug delivery systems (SMEDDSs) form translucent micro-emulsions with droplet size of less than $200 \mathrm{~nm}$. However, self nanoemulsifying drug delivery systems (SNEDDS) produces clear or transparent emulsion with droplets size less than $100 \mathrm{~nm}$ [30,31]. When compared with emulsions, which are sensitive and metastable dispersed forms, SEFs are physically stable formulations that are easy to manufacture. Thus, for lipophilic drug compounds that exhibit dissolution rate-limited absorption, these systems may offer an improvement in the rate and extent of absorption and result in more reproducible blood time profiles [25]. SEDDS are prepared in two forms: Liquid and solid SEDDS (S-SEDDS). S-SEDDS are prepared by solidification of liquid self-emulsifying components into powder. This powder is then used to produce various solid dosage forms, for example self-emulsifying pellets, self-emulsifying tablets etc [27]. S-SEDDS do not suffer with the problems like liquid SEDDS (L-SEDDS).

It has the advantages like:

Low manufacturing cost

More stability

More patient compliance because they are available as solid dosage form in tablets or pellet form.

In many studies it have been reported that SEDDS are used for delivering and targeting hydrophobic drugs such as coenzyme Q10, halofantrine, vitamin $\mathrm{E}$ and cyclosporine-A [27]. The solid SEDDS focus on the incorporation of liquid/semisolid ingredients into powders employing diverse solidification techniques like spray drying, melt granulation, moulding, melt extrusion, and nanoparticle technology. The powders can then be formulated as solid dosage forms like self emulsifying tablets and self-emulsifying pellets [24]. The idea of blending the potential SEDDS with that of the pellets through the inclusion of a self-emulsifying mixture into microcrystalline cellulose, and the production of pellets using extrusion-spheronization was first introduced by Newton, et al. [32].

High levels of surfactant typically present in SEDDS formulations can invariably lead to severe GI side-effects. Hence, a new class of SEDDS formulations, i.e., supersaturable SEDDS (SSEDDS) has been designed to reduce the amount of surfactant by incorporating a water soluble polymeric precipitation inhibitor (PPI) [33]. Such formulations have been developed specifically to reduce the surfactant side-effects and achieve rapid absorption of poorly soluble drugs [24]. The system is intended to generate and maintain a metastable supersaturated state in vivo by preventing or minimizing the precipitation of the drug through the use of a suitable PPI. Super saturation is intended to increase the thermodynamic activity of the drug beyond its solubility limit, and therefore, to result in an increased driving force for transit into and across the biological barrier [34]. The SSEDDS formulations have been demonstrated to improve both the rate and extent of the oral absorption of poorly water-soluble drugs quite effectively $[33,35,36]$. The inclusion of cellulosic polymers in the S-SEDDS formulation tends to effectively suppress the precipitation of drugs [37]. Various viscosity grades of Hydroxypropyl Methylcellulose (HPMC) are well-recognized for their ability to inhibit crystallization and, thereby, generate and maintain their supersaturated state for extended time periods [24]. In vitro dilution of the S-SEDDS formulation 


\section{Open Access Journal of Pharmaceutical Research}

results in the formation of a Micro emulsion, followed by slow crystallization of the drug on standing indicating that the supersaturated state of the system is prolonged by HPMC in the formulations. In the absence of HPMC, the SEDDS formulation undergoes rapid precipitation; yielding a lower drug concentration [33]. The significantly reduced amount of surfactant used in the S-SEDDS formulation approach significantly reduces toxicity and improves safety profile over the conventional SEDDS formulations [24].

Positively charged SEDDS have also been produced; many physiological studies have proved that the apical potential of absorptive cells, as well as that of all other cells in the body, is negatively charged with respect to the mucosal solution in the lumen. The drug exposure of the positively charged SEDDS has been found to be higher as well as the conventional formulations especially for bioavailability enhancement. The binding of the cationic SEDDS has been found to be much higher compared with anionically charged formulation, suggesting increased adhesion of the droplets to the cell surface due to electrostatic attraction [24]. Different dosage forms of SSEDDS include the dry emulsions, self-emulsifying capsules, self emulsifying sustained/controlled-release tablets, self-emulsifying sustained/controlled release pellets, self-emulsifying solid dispersions, self-emulsifying beads, self-emulsifying sustained-release microspheres, self-emulsifying nanoparticles, self-emulsifying suppositories and self-emulsifying implant [34].

\section{Advantages of SEDDS}

SEDDS possess the following advantages among others: [33]

Improvement and reduction in the variability of GI absorption of poor water soluble, lipophilic drugs.

Possible reduction in, or elimination of, a number of development and processing steps (e.g. salt selection or identification of a stable crystalline form of the drug, coating, taste masking, and reduced need for containment and clean-up requirements during manufacture of highlypotent or cytotoxic drug products).

Food does not interfere with the absorption of drug by use of such systems.

Relative ease of manufacture using readily available equipment.

The dose ranging from less than $25 \mathrm{mg}$ to greater than $2000 \mathrm{mg}$ can be administered by using these systems.

These systems enhance oral bioavailability due to bypass of hepatic metabolism and delivers drug directly into systemic circulation.
Inhibition of p-glycoprotein mediated drug efflux and preabsorptive metabolism by gut membrane bound cytochrome enzyme.

Protection of sensitive drug substances

High drug payloads.

Liquid or solid dosage forms.

Reduced energy requirement for emulsion formation.

Control of delivery profile

Promotion of lymphatic drug transport.

They enhance absorption of lipophilic drugs by stimulating pancreatic and biliary secretions and by prolongation of gastric residence time.

\section{Disadvantages of SEDDS}

Disadvantages of SEDDS include:

Lack of good predicative in vitro models for assessment of the formulations.

Traditional dissolution methods do not work, because formulations are independent on digestion prior to release of the drug.

In vitro model needs further development and validation.

Different prototype lipid based formulations needs to be developed and tested in vivo.

Chemical instabilities of drugs and high surfactant concentrations in formulations (approximately30-60\%) may irritate GIT.

Volatile co solvents may migrate into the shells of soft or hard gelatin capsules, resulting in the precipitation of the lipophilic drugs.

The precipitation tendency of the drug on dilution may be higher due to the dilution effect of the hydrophilic solvent. Formulations containing several components become more challenging to validation $[33,34]$.

\section{Self Nanoemulsifying Drug Delivery Systems (SNEDDSs)}

Self nanoemulsifying drug delivery systems (SNEDDS) are isotropic mixtures of oil, surfactant, co-surfactant and drug that form fine oil-in-water Nanoemulsion when introduced into aqueous phases under gentle agitation. SNEDDS spread readily in the gastrointestinal tract, and the digestive motility of the stomach and the intestine provide the agitation necessary for self-emulsification [35]. SEDDSs typically produce emulsions with turbid appearance, and droplet size between $200 \mathrm{~nm}$ to $5 \mu \mathrm{m}$, while self micro emulsifying drug delivery systems (SMEDDSs) form translucent micro-emulsions with droplet size of less than $200 \mathrm{~nm}$. However, self nanoemulsifying drug delivery systems (SNEDDS) produce clear or transparent emulsion with droplets size less than $100 \mathrm{~nm}[31,32]$. 


\section{Open Access Journal of Pharmaceutical Research}

Successful formulation of SNEDDS depends on the thorough understanding of the spontaneous nanoemulsification process and also on the physicochemical and biological properties of the components used for the fabrication of SNEDDS. The factors influencing the phenomenon of self nano-emulsification are:

The physicochemical nature and concentration of oily phase, surfactant and co-emulsifier or co surfactant or solubilizer (if included)

The ratio of the components, especially oil-surfactant ratio

The temperature and $\mathrm{pH}$ of the aqueous phase where nano-emulsification would occur

Physicochemical properties of the drug, such as hydrophilicity/lipophilicity, pKa and polarity.

These factors should receive attention while formulating SNEDDS. In addition, the acceptability of the SNEDDS components for the desired route of administration is also very important while formulating SNEDDS [35].

SNEDDS offer a reduction in bioavailability and can offer reproducibility in plasma profiles of drugs. The ability of the SNEDDS in improving $\mathrm{C}_{\max }$ and oral bioavailability or therapeutic effect has been established for various hydrophobic drugs. The improvement in bioavailability can be translated into reduction in the drug dose and dose-related side effects of many hydrophobic drugs, such as antihypertensive and anti-diabetic drugs [36]. Transretinol acetate SNEDDS emulsion, anti hyperlipidemic, Probucol, Estrogen receptor antagonist, Tamoxifencitrate, calcium channel blocker, Felodipine, and beta blocker, Carvedilol have been formulated as SNEDDS (35). SNEDDS are used for enhancing the solubility of anti-inflammatory drugs such as Indomethacin [37]. Fibrinolytic drugs such as Simvastatin, Atorvastatin, Valsartan, Gemfibrozil were also formulated as SNEDDS for improved bioavailability. Super-SNEDDS of Simvastat in show increased bioavailability compared to the conventional SNEDDS due to the increased drug loading [38]. Solid SNEDDS of Valsartan enhanced the bioavailability potential due to the presence of porous carriers and also showed stability for about six months which is an important factor [39]. Hormones such as Ondaster on hydrochloride and Insulin were also delivered orally by using SNEDDS. Solid SNEDDS of on dasteron showed increased bioavailability than the pure drug [40]. Insulin was formulated into SNEDDS by first forming insulin-phospholipid complex (IPC) and this was used as oil phase in the formulation. This showed good hypoglycemic effect in diabetic Wistar rats for oral administration. Hence IPC can be used for the oral delivery of Insulin [41].
Anti-cancer drugs were also formulated as SNEDDS. They include Raloxifene hydrochloride, Cyclosporine A, Paclitaxel, Flutamide. In Raloxifene, the uptake of the drug by endocrine organs was assessed by administering the SNEDDS in alkalinized and non-alkalinized form to Wistar rats. Non-alkalinized form showed good uptake by the endocrine organs than the alkalinized form [42]. SNEDDS pellets of cyclosporine A were formulated by fluid bed coating technique and this improved the in vivo performance of the drug [43]. The drug release profile of Paclitaxel was improved by SNEDDS and the dissolution rate was also faster compared to that of the pure drug in Flutamide $[36,44,45]$.

SNEDDS are given in the form of soft or hard gelatin capsules. They reach the gastro intestinal tract and the GI motility of the stomach provides the agitation for selfemulsification. Because of this self-emulsification the drug is given as small droplets with size less than $5 \mu \mathrm{m}$ for improved solubility. After administering orally, lingual and pancreatic lipases act on the oily phase of the SNEDDS that result in the formation of emulsified monoglycerides, di-glycerides and fatty acids. This in the presence of bile acids leads to the formation of intestinal mixed micelles. When these mixed micelles pass through the enterocytes, it leads to the formation of chylomicrons. These drain the drug into the lymphatic vessels and not in the blood vessels thus bypassing the first pass effect. Thus the oral bioavailability gets increased [36].

\section{Advantages of SNEDDS}

Advantages of SNEDDS include:

Protection of sensitive drug substances.

Selective targeting of drug(s) toward specific absorption window in GIT.

Enhanced oral bioavailability enabling reduction in dose. High drug payloads.

It can be easily stored since it belongs to a thermodynamics stable system.

Fine oil droplets would pass rapidly and promote wide distribution of the drug throughout the GIT, thereby minimizing the irritation frequently encountered during extended contact between bulk drug substance and the gut wall.

As compared with oily solutions, they provide a large interfacial area for partitioning of the drug between oil and water [35].

\section{Disadvantages of SNEDDS}

Disadvantages of SNEDDS include:

Lack of good predicative in vitro models for assessment of the formulations because traditional dissolution methods do not work, because these formulations potentially are 


\section{Open Access Journal of Pharmaceutical Research}

dependent on digestion prior to release of the drug. To mimic this, an in vitro model simulating the digestive processes of the duodenum has been developed.

Need of different prototype lipid based formulations to be developed and tested in vivo in a suitable animal model [35].

\section{Factors affecting SNEDDS}

There are many factors that affect SNEDDS:

Drugs which are administered at very high dose are not suitable for SNEDDS, unless they exhibit extremely good solubility in at least one of the components of SNEDDS, preferably lipophillic phase. The drugs exhibit limited solubility in water and lipids are most difficult to deliver by SNEDDS.

The ability of SNEDDS to maintain the drug in solubilized form is greatly influenced by the solubility of the drug in oily phase. If the surfactant or co-surfactant is contributing to a greater extent for drug solubilization, then there could be a risk of precipitation, as dilution of SNEDDS will lead to lowering of solvent capacity of surfactant or co-surfactant [35].

\section{Solid Self-Nanoemulsifying Drug Delivery Systems (SSNEDDSs)}

Solid SNEDDS was developed in order to eliminate the disadvantages associated with liquid SNEDDS handling, manufacturing and stability. Solid SNEDDS in the form of dry, solid powders would help in overcoming the limitations associated with liquid SNEDDS. Solid dosage forms are most stable and are convenient for handling; therefore, attempts are made to convert the liquid systems into solid SNEDDS. Various techniques, such as spray drying, freeze drying and adsorption on carriers, can be employed to convert liquid SNEDDS into solid SNEDDS compressed into tablets. The selection of a particular process for preparation of solid SNEDDS would depend on the content of oily excipients of the formulation, properties of active pharmaceutical ingredients, such as solubility, heat stability and compatibility with other ingredients [46].

The simplest technique to convert liquid SNEDDS to solid SNEDDS is by adsorption onto the surface of carriers or by granulation using liquid SNEDDS as a binder. This technique is uncomplicated, cost effective, easily optimized and industrially scalable. It can be used for heat-and moisture-sensitive molecules, thus providing an advantage over other techniques, such as spray drying and freeze drying. Various excipients utilized for the preparation of solid oral dosage forms can be employed for adsorption. The excipients should possess large surface areas to adsorb sticky and sometimes viscous oily SNEDDS formulation [46].

The ability of different excipients, such as dibasic calcium phosphate, lactose, micro crystalline cellulose, colloidal silicon dioxide and Neusilin, to adsorb cefpodoxime proxetil SNEDDS have been studied [46]. Solidification techniques for converting liquid/semisolid SEDDS/SNEDDS to solids include:

a) Capsule filling with liquid and semisolid selfemulsifying formulations: Capsule filling is the simplest and the most common technology for the encapsulation of liquid or semisolid SE formulations for the oral route. The advantages of capsule filling are simplicity of manufacturing, suitability for low-dose highly potent drugs and high drug loading up to $50 \%(\mathrm{w} / \mathrm{w})$ potential [47].

b) Spray drying: This technique involves the preparation of a formulation by mixing lipids, surfactants, drug, solid carriers, and solubilization of the mixture before spray drying. The solubilized liquid formulation is then atomized into a spray of droplets. The droplets are introduced into a drying chamber, where the volatile phase (e.g. the water contained in 7anemulsion) evaporates, forming dry particles under controlled temperature and airflow conditions. Such particles can be further prepared into tablets or capsules [47].

c) Spray cooling: Spray cooling also referred to as spray congealing is a process whereby the molten formula is sprayed into a cooling chamber. Upon contact with the cooling air, the molten droplets congeal and re-crystallize into spherical solid particles that fall to the bottom of the chamber and subsequently collected as fine powder. The fine powder may then be used for development of solid dosage forms, tablets or direct filling into hard shell capsules. Many types of equipment are available to atomize the liquid mixture and to generate droplets: rotary pressure, two-fluid or ultrasonic atomizers [47,49].

d) Adsorption to solid carriers: SEDDS can be adsorbed at high levels (up to $70 \%(\mathrm{w} / \mathrm{w})$ ) onto suitable carriers. Solid carriers can be microporous inorganic substances, high surface area colloidal inorganic adsorbent substances, cross-linked polymers or nanoparticle adsorbents (eg., silica, silicates, magnesium trisilicate, magnesium hydroxide, talcum, crospovidone, cross-linked sodium carboxymethyl cellulose and cross linked polymethyl methacrylate).The adsorption technique has been successfully applied to gentamicin and erythropoietin with caprylocaproylpolyoxyl glycerides 


\section{Open Access Journal of Pharmaceutical Research}

(Labrasol) formulations that maintained their bioavailability enhancing effect after adsorption on carriers [50-52].

e) Melt granulation: Melt granulation or pelletization is a one step-process allowing the transformation of a powder mix (containing the drug) into granules or spheronized pellets. The technique needs high shear mixing in presence of a meltable binder. This is referred to as "pump-on" technique. Alternatively, the binder may be blended with the powder mix in its solid or semi-solid state and allowed to melt (partially or completely) by the heat generated from the friction of particles during high shear mixing referred to as "melt-in" process. The melted binder forms liquid bridges with the powder particles that shape into small agglomerates (granules) which can, by further mixing under controlled conditions transform to spheronized pellets [47].

f) Melt extrusion/Extrusion spheronization: It is a solvent-free process that allows high drug loading (60\%) as well as content uniformity. Applying extrusionspheronization, SE pellets of diazepam and progesterone and bi-layered cohesive SE pellets have been prepared $[53,54]$.

\section{Advantages of Nanoemulsion drug delivery system}

The advantages of Nanoemulsions drug delivery systems include [55-57]

The small size of the droplets allows them to deposit uniformly on substrates. Wetting, spreading and penetration may be also enhanced as a result of the low surface tension of the whole system and the low interfacial tension of the $\mathrm{o} / \mathrm{w}$ droplets.

The very small droplet size causes a large reduction in the gravity force and the Brownian motion may be sufficient for overcoming gravity. This means that no creaming or sedimentation occurs on storage.

The small droplet size also prevents any flocculation of the droplets. Weak flocculation is prevented and this enables the system to remain dispersed with no separation. Nanoemulsions are thermodynamically stable system and the stability allows self emulsification of the system.

The small droplets also prevent their coalescence, since these droplets are elastic, surface fluctuations are prevented.

Nanoemulsions are suitable for efficient delivery of active ingredients through the skin. The large surface area of the emulsion system allows rapid penetration of actives. It is non-toxic and non-irritant so can be easily applied to skin and mucous membranes.
The transparent nature of the system, their fluidity (at reasonable oil concentrations) as well as the absence of any thickeners may give them a pleasant aesthetic character and skin feel.

Unlike micro emulsions (which require a high surfactant concentration, usually in the region of $20 \%$ and higher), Nanoemulsions can be prepared using reasonable surfactant concentration. For a $20 \%$ o/w Nanoemulsion, a surfactant concentration in the region of $5-10 \%$ may be sufficient. Nanoemulsions are usually formulated with surfactants, which are approved for human consumption (GRAS), they can be taken by enteric route.

Nanoemulsions can be applied for delivery of fragrance, which may be incorporated in many personal care products. This could also be applied in perfumes, which are desirable to be formulated alcohol free.

Nanoemulsions may be applied as a substitute for liposome and vesicles (which are much less stable) and it is possible in some cases to build lamellar liquid crystalline phases around the Nanoemulsion droplets.

Nanoemulsions can be formulated in numerous dosage forms such as creams, liquids, sprays and foams.

They do not damage healthy human and animal cells, so nano emulsions are suitable for human and veterinary therapeutic purposes.

Increase the rate of absorption, increases bioavailability and eliminates variability in absorption

Helps solubilize lipophilic drug and masks unpleasant taste of some drugs

Various routes like topical, oral and intravenous can be used to deliver the product.

Better uptake of oil-soluble supplements in cell cultures. Improve growth and vitality of cultured cells. It allows toxicity studies of oil-soluble drugs in cell cultures.

Nanoemulsions could enhance the stability of chemically unstable compounds by protecting them from oxidative degradation and degradation by light.

Possibilities of controlled drug release and drug targeting, and the incorporation of a great variety of therapeutic actives.

\section{Major challenges}

Although Nanoemulsions provide great advantages as a delivery system, however they suffer for some major challenges and limitations which include: [55-57]

The formulation of Nanoemulsions is an expensive process due to size reduction of droplets is very difficult as it required a special kind of instruments and process methods. For example, homogenizer (instrument required for the Nanoemulsions formulation) arrangement is an expensive process. More ever microfluidization and ultra sonication (manufacturing process) require large amount of financial support. 


\section{Open Access Journal of Pharmaceutical Research}

One problem associated with Nanoemulsion is their stability. Although it is generally accepted that these systems could remain stable even by years, however, due to the small droplet size, it has been reported that the Oswald ripening could damage Nanoemulsions, causing their application to be limited. Therefore, in most cases, Nanoemulsions are required to be prepared shortly before their use.

Use of a large concentration of surfactant and co surfactant necessary for stabilizing the nanodroplets.

Limited solubility capacity for high melting substances.

Nanoemulsion stability is influenced by environmental parameters such as temperature and $\mathrm{pH}$.

Lack of understanding of the mechanism of production of submicron droplets and the role of surfactants and co surfactants.

Lack of demonstration of the benefits that can be obtained from using Nanoemulsions when compared with the classical macro emulsion systems.

Lack of understanding of the interfacial chemistry that is involved in production of Nanoemulsions.

\section{Formulation of Nanoemulsions}

Materials used in preparation of Nanoemulsions: Nanoemulsions are prepared using oils, surfactants and co-surfactants and aqueous phase; [58]. Oils used in Nanoemulsions preparation include Captex 355, Captex 8000, Witepsol, Myritol 318, Isopropyl myristate, Capryol 90, Sefsol-218, triacetin, isopropyl myristate, castoroil, olive oil, etc. Solubility of the drug in the oil phase is an important criterion for the selection of oils. This is particularly important in the case of oral formulation development, as the ability of Nanoemulsion to maintain the drug in solubilized form is greatly influenced by the solubility of the drug in the oil phase. While water-in-oil Nanoemulsions are better choice for hydrophilic drugs lipophilic drugs are preferably solubilized in oil-in-water Nanoemulsions. Drug loading in the formulation is a very critical design factor in the development of Nanoemulsions for poorly soluble drugs, which is dependent on the drug solubility in various formulation components. An understanding of factors influencing drug loading capacity while maintaining the capability of the system to undergo monophasic dilution with water and minimizing the tendency for drug precipitation or crystallization in diluted systems is essential to the design of stable and appropriately low-volume Nanoemulsion systems for drug delivery applications [59,64]. Edible oils are not frequently useful due to their poor ability to dissolve large amounts of lipophilic drugs. Moreover, formulation of Nanoemulsion with oil of low drug solubility would require incorporation of more oil to incorporate the target drug dose, which in turn would require higher surfactant concentration to achieve oil solubilization, which might increase the toxicity of the system. Novel semi-synthetic medium chain derivatives (as amphiphilic compounds) having surfactant properties are progressively and effectively replacing the regular medium chain triglyceride oils $[65,66]$.

Surfactants used for stabilizing Nanoemulsions may be non ionic, zwitterionic, cationic and an ionic. The surfactants may include Capryol 90, Gelucire 44/14, 50/13, Cremophor RH 40,Imwitor 191, 742, 780 k, 928, 988, Labrafil CS, M, 2125 CS, Lauroglycol 90, PEG MW > 4000,PlurolOleique CC 497, Poloxamer 124 and 188, Softigen 701, 767, Labrasol, Cremophor EL, Tween 20, Tween 60, and Tween 80, etc. Components of Nanoemulsion-based systems are associated with toxicity concerns. Large amounts of surfactants may cause gastrointestinal and skin irritation when administered orally and topically, respectively. Therefore, the proper selection of surfactants is essential. Rational use of the minimum concentration of the surfactant in the formulation is advocated. Nonionic surfactants are relatively less toxic than their ionic counter parts and typically have lower critical micelle concentration (CMCs). Also, o/w Nanoemulsion dosage forms for oral or parenteral use based on nonionic surfactants are likely to offer in vivo stability [67]. Therefore, proper selection of surfactants is a crucial factor.

Another important criterion is the selection of surfactant with proper hydrophile-lipophile balance (HLB) value. Hydrophilic surfactants and co-surfactants are considered to prefer the interface and to lower the necessary energy to form the Nanoemulsions, thereby improving the stability. For instance, the required HLB value to form o/w Nanoemulsion is greater than 10 [68]. The right blend of low and high HLB surfactants leads to the formation of a stable Nanoemulsion upon dilution with water. The type and nature of the surfactant is also an important factor for consideration; nonionic surfactants are usually selected since they are known to be less affected by $\mathrm{pH}$ and changes in ionic strength, are generally regarded as safe, and are biocompatible; ionic surfactants are less commonly used due to toxicological concerns. Solubilization of oil with the surfactant is also an important factor. It is not necessary that the same surfactant that has good solubilizing power for drugs would have equally good affinity for the oil phase. Surfactant-oil miscibility can thus give an initial indication on the possibility of Nanoemulsion formation with this system. 


\section{Open Access Journal of Pharmaceutical Research}

Co surfactants are added to obtain Nanoemulsion systems at low surfactant concentration. Short-to medium-chain-length alcohols (C3-C8) are commonly added as co surfactants, which further reduce the interfacial tension and increase the fluidity of the interface $[70,71]$. They also increase the mobility of the hydrocarbon tail and allow greater penetration of the oil into this region. Alcohols may also increase the miscibility of the aqueous and oily phases due to its partitioning between these phases. Co-surfactants used in Nanoemulsions include TranscutolP, glycerin, ethyleneglycol, ethanol, propanol, ethanol, isopropyl alcohol, n-butanol, PEG 400, Carbitol, and propylene glycol. Nanoemulsion area is often used as the assessment criterion for the evaluation of co surfactants. The larger the size of the Nanoemulsion field, the greater the nanoemulsification efficiency of the system.

Moreover, the most important criterion for selection of all the Nanoemulsion components is that all the excipients should be pharmaceutically acceptable for oral administration or topical application, etc., depending upon the requirement and falling under GRAS category.

\section{Methods of Preparation of Nanoemulsions}

As Nanoemulsions are non-equilibrated systems, and so their preparation involves the input of a large amount of either energy or surfactants and in some cases a combination of both. As a result, high energy or low energy methods can be used in their formulation. Although high energy emulsification method is traditionally used for the preparation of Nanoemulsion formulation but low emulsion emulsification method now create an attraction due to their wide application and advantages as a formulation and stability aspects. Generally, energy is usually required in emulsion formulation because the process may be non-spontaneous [72-74].

The production of Nanoemulsions costs more energy than that required to produce Macroemulsion. Presence of surfactants help lower the surface tensions between oil and water. Small molecules such as non-ionic surfactants lower surface tension more than polymeric surfactants such as poly (vinyl alcohol). Another important role of the surfactant is its effect on the interfacial dilatational modulus. During emulsification an increase in the interfacial area takes place and this causes a reduction in surface excess. The equilibrium is restored by adsorption of surfactant from the bulk, but this takes time (shorter times occur at higher surfactant activity). Because of the lack or slowness of equilibrium with polymeric surfactants, dilatational modulus will not be the same for expansion and compression of the interface. In practice, surfactant mixtures are used and these have pronounced effects on surface tension and dilatational modulus [76]. Some specific surfactant mixtures give lower surface tension values than either of the two individual components. Polymer-surfactant mixtures may show some synergistic surface activity. An important role of the emulsifier is to prevent shear induced coalescence during emulsification. The requirement is that the continuous phase has a significant excess of surfactant. This excess enables new surface area of the nano-scale droplets to be rapidly coated during emulsification; thereby inhibiting shear-induced coalescence. This excess is generally in the form of surfactant micelles in the continuous phase. These micelles dissociate into monomers that rapidly adsorb onto the surfaces of newly created droplets [74].

\section{Low Energy Methods}

As the name suggests, low-energy emulsification methods require low energy for the fabrication of Nanoemulsions. These methods are mainly dependent on modulation of interfacialphenomenon/phase transitions and intrinsic physicochemical properties of the surfactants, coemulsifiers/co-surfactants and oil to yield nano-sized emulsion droplets. The lower energy method, also called the condensation method, is based on the phase transitions taking place during the emulsification process $[76,77]$. These phase transitions result from changes in the spontaneous curvature of the surfactant and can be achieved-

I. At constant composition by changing the spontaneous curvature of non-ionic surfactants with temperature, the well known Phase Inversion Temperature, PIT, widely used in industry or

II. At constant temperature by varying the composition of the system by the Emulsion Inversion Point (EIP) method [78-80].

In other words, low-energy emulsification method was developed according to the phase behavior and properties of the constituents, to promote the formation of ultra small droplets. These low energy techniques include selfemulsification, phase transition and phase inversion temperature methods. The low energy method is interesting because it utilizes the stored energy of the system to form small droplets. This emulsification can be brought about by changing the parameters which would affect the hydrophiliclipophilic balance (HLB) of the system like temperature, composition, etc. The limitations include complexity, precise approach required and use of 


\section{Open Access Journal of Pharmaceutical Research}

synthetic surfactants. Ina nutshell, the most commonly used low-energy emulsification methods include:

Phase Inversion Temperature (PIT) method: This method employs temperature-dependent solubility of non-ionic surfactants, such as polyethoxylated surfactants, to modify their affinities for water and oil as a function of the temperature. It has been observed that polyethoxylated surfactants tend to become lipophilicon heating owing to dehydration of polyoxyethylene groups [81]. This phenomenon forms a basis of Nanoemulsion fabrication using the PIT method. In the PIT method, oil, water and nonionic surfactants are mixed together at room temperature. This mixture typically comprises $\mathrm{o} / \mathrm{w}$ micro emulsions coexisting with excess oil, and the surfactant monolayer exhibits positive curvature. When this macro emulsion is heated gradually, the polyethoxylated surfactant becomes lipophilic and at higher temperatures, the surfactant gets completely solubilized in the oily phase and the initial o/w emulsion undergoes phase inversion to $\mathrm{w} / \mathrm{o}$ emulsion. The surfactant monolayer has negative curvature at this stage. At an intermediate temperature (also termed HLB temperature), the non-ionic surfactant has similar affinity for aqueous and oily phase, and this ternary system has extremely low interfacial tension (in the order of 10-210-5 mNm-1) and spontaneous curvature typically reaches zero. The ternary system at this stage typically consists of a D-phase bicontinuous Micro emulsion or a mixture of a D phase bicontinuous micro emulsion and lamellar liquid crystalline phases. It has been observed that Nanoemulsions with very small droplet size and polydispersity index can be generated by rapid cooling of the single-phase or multiphase bicontinuous micro emulsions maintained at either PIT or a temperature above PIT (transitional-phase inversion). Nanoemulsions can also be generated by rapidly diluting the single bicontinuous micro emulsions with the aqueous or oil phase (catastrophic phase inversion) to obtain either $\mathrm{o} / \mathrm{w}$ Nanoemulsion orw/o Nanoemulsion. It has been observed that the characteristics of the Nanoemulsion are mainly dependent on the structure of the surfactant at HLB temperature (bicontinuous orlamellar) and also on the surfactant/oil ratio. Initially, PIT method was believed to be useful for fabricating o/w Nanoemulsions. However, in recent years, the application of the PIT method has been established for fabricating w/o emulsions and Nanoemulsions. It is important to note that the use of lipophilic polyethoxylated surfactants and appropriate modifications in the typical PIT protocol are required for obtaining w/o Nanoemulsions.
Solvent displacement method: The solvent displacement method for spontaneous fabrication of Nanoemulsion has been adopted from the nanoprecipitation method used for polymeric nanoparticles. In this method, oily phase is dissolved in water-miscible organic solvents, such as acetone, ethanol and ethylmethyl ketone. The organic phase is poured into an aqueous phase containing surfactant to yield spontaneous Nanoemulsion by rapid diffusion of organic solvent. The organic solvent is removed from the Nanoemulsion by a suitable means, such as vacuum evaporation [82-84].

Phase Inversion Composition Method (SelfNanoemulsification Method): This method generates Nanoemulsions at room temperature without use of any organic solvent and heat. Forgirani et al. observed that kinetically stable Nanoemulsions with small dropletsize $(\sim 50 \mathrm{~nm})$ can be generated by the stepwise addition of water into solution of surfactant inoil, with gentle stirring and at constant temperature. Although the components used in the fore mentioned investigation were not of pharmaceutical grade, the investigation openeddoors to design pharmaceutically acceptable Nanoemulsions using a similar approach. The spontaneous nano-emulsification has been related to the phase transitions during the emulsification process and involves lamellar liquid crystalline phases or D-type bicontinuous microemulsion during the process [80].

\section{High Energy Methods}

High-energy emulsification methods make use of devices that use very high mechanical energy to create Nanoemulsions with high kinetic energy. The high-energy method utilizes mechanical devices to create intensely disruptive forces which break up the oil and water phases to form nano-sized droplets. This can be achieved with ultrasonicators, microfluidiser and high pressure homogenizers [84]. Particle size here will depend on the type of instruments employed and their operating conditions like time and temperature along with sample properties and composition. These methods include highpressure homogenization and ultrasonic emulsification. High-pressure homogenization is the most common method used for the fabrication of Nanoemulsions [82]. During high-pressure homogenization, the coarse macroemulsion is passed through a small orifice at an operating pressure in the range of 500 to 5000 psi. During this process, several forces, such as hydraulic shear, intense turbulence and cavitations, act together to yield Nanoemulsions with extremely small droplet size. The resultant product can be re-subjected to high-pressure homogenization until Nanoemulsion with desired droplet size and polydispersity index is obtained. Micro- 


\section{Open Access Journal of Pharmaceutical Research}

fluidization employs a high-pressure positive displacement pump operating at very high pressures, up to 20,000 psi. This pump forces macroemulsion droplets through the interaction chamber consisting of a series of micro-channels. The macroemulsion flowing through the micro channels collides with high velocity on to an impingement area resulting in very fine Nanoemulsions.

The Nanoemulsions with desired size range and dispersity can be obtained by varying the operating pressure and the number of passes through interaction chambers like high pressure homogenization. Ultrasonic emulsification uses a probe that emits ultrasonic waves to disintegrate the macroemulsion by means of cavitations forces. By varying the ultrasonic energy input and time, the Nanoemulsions with desired properties can be obtained [87].

High-energy emulsification methods can be employed to fabricate both $\mathrm{o} / \mathrm{w}$ and $\mathrm{w} / \mathrm{o}$ Nanoemulsions. Highpressure homogenization and micro fluidization can be used for fabrication of Nanoemulsions at laboratory and industrial scale, whereas ultrasonic emulsification is mainly used at laboratory scale. In addition, high-energy methods require sophisticated instruments and extensive energy input, which considerably increases the cost of Nanoemulsions fabrication [85].

This is particularly significant in the pharmaceutical sciences. High energy methods allow for a greater control of particle size and a large choice of composition, which in turn controls the stability, rheology and colour of the emulsion. Although high-energy emulsification methods yield Nanoemulsions with desired properties and have industrials capability, they may not be suitable for thermo-labile drugs such as retinoid and macromolecules, including proteins, enzymes and nucleic acids. Moreover, high energy methods alone normally do not yield oil droplets $(<100 \mathrm{~nm})[85,86]$.

\section{Formulation Factors that Affect the Stability of Nanoemulsions}

Although Nanoemulsions enhance the physical as well as chemical stability of drugs, stability of drug product is one of the problems associated with the development of Nanoemulsions $[88,91]$. Stability studies are performed on Nanoemulsions by storing them at refrigerator and room temperatures over a number of months. The viscosity, refractive index and droplet size are determined during this period of storage. Insignificant changes in these parameters indicate formulation stability. Accelerated stability studies can also be performed on the Nanoemulsions. In this instance, Nanoemulsion formulation are kept at accelerated temperatures and samples withdrawn at regular intervals and analyzed for drug content by stability indicating assay methods. The amount of drug degraded and remaining in Nanoemulsion formulation is determined at each time interval $[90,92]$. Stability of Nanoemulsion formulation may be enhanced by controlling factors such as type and concentration of surfactant and co surfactant, type of oil phase, methods used, process variables and addition of additives. Overall Nanoemulsion formulation may be considered as effective, safe and patient compliance formulation for the delivery of pharmaceuticals.

Factors to be considered during preparation of Nanoemulsion include the following among others: [93]

a. The prime requirement in Nanoemulsion production is that an ultra low interfacial tension should be attained at the oil water interface, so surfactants must be carefully chosen.

b. Concentration of surfactant must be high enough to provide the number of surfactant molecules needed to stabilize the nano droplets.

c. The interface must be flexible to promote the formation of Nanoemulsion.

\section{Characterization of SNEDDS}

Ternary phase diagrams: Construction of ternary or pseudo-ternary phase diagrams is usually employed in the development of SEDDS. Ternary phase diagrams enable comparison of different surfactants and their synergistic effect with co-surfactant. They can also help to determine the optimum concentration ranges of different excipients and to identify the self-emulsification regions. The boundaries of different phase regions can easily be assessed visually.

Emulsification time: With the purpose of quantifying the efficiency of emulsification of SEDDS, P out on employed the rotating paddle to promote emulsification in a crude nephelometer. This enabled an estimation of the time taken for emulsification. On completion of emulsification, the SEDDS samples were taken for particle sizing by photon correlation spectroscopy, and further by other characterizations.

Droplet Size Analysis: The droplet size of the nanoemulsion was determined by photon correlation spectroscopy. The formulation $(0.1 \mathrm{~mL})$ was dispersed in $50 \mathrm{~mL}$ of water in a volumetric flask and gently mixed by inverting the flask. Measure by using a Zetasizer and light scattering monitor at $25^{\circ} \mathrm{C}$ at $90^{\circ}$ or $180^{\circ}$ angle [60].

Transmission Electron Microscopy: Morphology and structure of the Nanoemulsion usually determined by 


\section{Open Access Journal of Pharmaceutical Research}

transmission electron microscopy (TEM). A combination of bright-field imaging at increasing magnification and of diffraction modes use to reveal the form and size of the Nanoemulsion. To perform the TEM observations, the nanoemulsion formulation dilute with water $(1 / 100)$. A drop of the diluted Nanoemulsion directly deposited on the film grid and observed after dry $[61,62]$.

Refractive Index: The refractive index, $n$, of a medium is defined as the ratio of the speed c, of a wave such as light or sound in a reference medium to the phase speed, vp, of the wave in the medium represented by equation;

$\mathrm{n}=\mathrm{c} / \mathrm{vp}$

It was determined using an Abbes type Refractrometer $[60,61]$.

Drug Content: Drug content determine by reverse phase HPLC method using different columns of appropriate porosity $[61,63]$.

\section{Future Perspectives}

Nanoemulsions are proposed for numerous applications in pharmacy as drug delivery systems because of their capacity to solubilize non-polar active compounds. Future perspectives of Nanoemulsion are very promising in different fields of therapeutics or application in development of cosmetics for hair or skin. One of the versatile applications of Nanoemulsions is in the area of drug delivery where they act as efficient carriers for bioactive, providing administration by various routes. The advantages and applications of Nanoemulsions for oral drug delivery are abundant, where the droplet size is related to their absorption in the gastrointestinal tract. Due to the renewed interest in herbal drug formulation, Nanoemulsion may be the ideal delivery platform for these difficult-to-formulate phytopharmaceuticals. The prospects of Nanoemulsions lie in the ingenuity of formulation experts to utilize the advantages of Nanoemulsion carriers in overcoming peculiar problems of drug delivery such as absorption, permeation and stability of both orthodox and herbal drugs.

\section{Conclusion}

Nanoemulsions offer several advantages for the delivery of drugs and are thus receiving growing attention as drug carriers for improving the delivery of active pharmaceutical ingredients. They are applicable for almost all routes of delivery and therefore hold promise for different fields, be it cosmetics, therapeutics or biotechnology. This new technology could be developed to overcome the poor absorption of some phytopharmaceuticals and poor miscibility of these compounds with the lipid contents of cell membrane linings.

\section{References}

1. Kotta S, Khan AW, Pramod K, Ansari SH, Sharma RK, et al. (2012) Exploring oral Nanoemulsions for bioavailability enhancement of poorly water-soluble drugs. Expert Opin Drug Deliv 9(5): 585-598.

2. Thi TD, Van Speybroeck M, Barillaro V, Martens J, Annaert P, et al. (2009) Formulate-ability of ten compounds with different physicochemical profiles in SMEDDS. Eur J Pharm Sci 38(5): 479-488.

3. Fatouros DG, Deen GR, Arleth L, Bergenstahl B, Nielsen FS, et al. (2007) Structural development of self nano emulsifying drug delivery systems (SNEDDS) during in vitro lipid digestion monitored by small-angle X-ray scattering. Pharm Res 24(10): 1844-1853.

4. Nazzal S, Smalyukh I, Lavrentovich O, Khan M (2002) Preparation and in vitro characterization of a eutectic based semisolid self nanoemulsified drug delivery system (SNEDDS) of ubiquinone: mechanism and progress of emulsion formation. Int J Pharm 235(12): 247-265.

5. Preshita P, Abhijit AD, Vandana BP (2002) Overcoming poor oral bioavailability using nanoparticle formulations opportunities and limitations. Drug Discov Today Technol 9: e87-e95.

6. Date A, Desai N, Dixit R, Nagersanker M (2010) Selfnanoemulsifying drug delivery systems: formulation insights, applications and advances. Nanomedicine (Lond) 5(10): 1595-1616.

7. Elkasabgy NA (2014) Ocular supersaturated selfnanoemulsifying drug delivery systems (S-SNEDDS) to enhance econazole nitrate bioavailability. Int J Pharm 460(1-2): 33-44.

8. Cuine JF, McEvoy CL, Charman WN, Pouton CW, Edwards GA, et al. (2008) Evaluation of the impact of surfactant digestion on the bioavailability of danazol after oral administration of lipidic self-emulsifying formulations to dogs. J Pharm Sci 97(2): 995-1012.

9. Balakumar K, Raghavan CV, Selvan NT, Prasad RH, Abdu S (2013) Self nanoemulsifying drug delivery system (SNEDDS) of rosuvastatin calcium: design, formulation, bioavailability and pharmacokinetic 


\section{Open Access Journal of Pharmaceutical Research}

evaluation. Colloids Surf B Biointerfaces 112: $337-$ 343.

10. Gursoy R, Benita S (2004) Self-emulsifying drug delivery systems (SEDDS) for improved oral delivery of lipophilic drugs. Biomed Pharmacother 58(3): 173182.

11. Ravi TPU, Padma T (2011) Nanoemulsions for drug delivery through different routes. Res. Biotechnol 2(3): 1-13.

12. Dixit RP, Nagarsenker MS (2008) Selfnanoemulsifying granules of ezetimibe: design, optimization and evaluation. Eur J Pharm Sci 35(3): 183-192.

13. Shafiq S, Shakeel F, Talegaonkar S, Ahmad FJ, Khar RK, et al. (2007) Development and bioavailability assessment of ramipril nanoemulsion formulation. Eur J Pharm Biopharm 66(2): 227-243.

14. Sutradhar K B, Amin M L (2013) Nanoemulsions: increasing possibilities in drug delivery. Eur J Nanomed 5(2): 97-110.

15. Hamed AC, Vitthal VC, Pravin DC (2013) Self emulsifying drug delivery system: A review. Int J Pharm Chem Sci 2(1): 34- 44.

16. Chudasama A, Patel V, Nivsarkar M, Vasu K, Shishoo C (2011) A novel lipid-based oral drug delivery system of nevirapine. Int J Pharm Tech Res 3(2):1159-1168.

17. Jingling $\mathrm{T}$, Jin S, Fude $\mathrm{C}$, Zhonggui $\mathrm{H}$ (2006) Preparation of self-emulsifying drug delivery systems of Ginkgo biloba extracts and in vitro dissolution studies. Asian J Trad Med 1: 3-4.

18. Chime SA, Onyishi VI (2013) Lipid-based drug delivery systems (LDDS): Recent advancesand applications of lipids in drug delivery. Afr J Pharm Pharmacol 7(48): 3034-3059.

19. Reiss H (1975) Entropy-induced dispersion of bulk liquids. J Colloid Interf Sci 53(1):61-70.

20. Constantinides PP (1995) Lipid microemulsions for improving drug dissolution and oral absorption: physical and biopharmaceutical aspects. Pharm Res 12(11): 1561-1572.

21. Dabros T, Yeung A, Masliyah J, Czarnecki J (1999) Emulsification through Area Contraction. J Colloid Interf Sci 210(1): 222-224.
22. Wakerly MG, Pouton CW, Meakin BJ, Morton FS (1986) Self-emulsification of vegetable oil-non-ionic surfactant mixtures. ACS Symp Series 311: 242-255.

23. Rang MJ, Miller CA (1999) Spontaneous emulsification of oils containing hydrocarbon, nonionic surfactant, and oleyl alcohol. J Colloid Interf Sci 209(1): 179-192.

24. Bhupinder S, Shantanu B, Rishi K, Ramandeep S, Katare OP (2009) Self-emulsifying drug delivery systems (SEDDS): Formulation development, characterization and applications. Crit Rev Ther Drug Carr Syst 26(5): 427-521.

25. Patel BR, Patel PR, Patel M (2008) Self- emulsifying drug delivery systems. Pharm Sci Tech 7: 1-3.

26. Rajan BM, Nirav SS (2011) A reviews: Self emulsifying drug delivery system. Int J Pharm Pharm Sci 3(2): 2328.

27. Shobhit K, Satish KG, Pramod KS (2012) Selfemulsifying drug delivery systems(sedds) for oral delivery of lipid based formulations - A review. Afr J Basic App Sci 4(1): 07-11.

28. Obitte NC, Chukwu A (2010) The excipient usefulness of Carbosil ${ }^{\circledR}$ and Landolphiaowariensis in two oilbased self-emulsifying formulations. Asian J Pharmaceut 4(4): 213-219.

29. Sachan R, Khatri K, Kasture SB (2010) Selfemulsifying drug delivery system a novel approach for enhancement of bioavalibility. Int J Pharm Tech Res 2(3): 1738-1745.

30. Balakumar K, Raghavan CV, Selvan NT, Prasad RH, Abdu S (2013) Self nanoemulsifying drug delivery system (SNEDDS) of rosuvastatin calcium: Design, formulation, bioavailability and pharmacokinetic evaluation. Colloids Surf Biointer 112: 337-343.

31. Wadhwa J, Nair A, Kumria R (2012) Emulsion forming drug delivery system for lipophilicdrugs, Acta Pol Pharm 69(2): 179-191.

32. Newton M, Petersson J, Podczeck F, Clarke A, Booth S (2001) The influence of formulation variables on the properties of pellets containing a self-emulsifying mixture. J Pharm Sci 90(8): 987-995.

33. Gajendra S, Khinchi MP, Gupta MK, Dilip A, Adil H, et al. (2012) Self emulsifying drug delivery systems 


\section{Open Access Journal of Pharmaceutical Research}

(SEEDS): An approach for delivery of poorly water soluble drug. Int J Pharm Life Sci 3(9): 1991-1996.

34. Sharma V, Saxena PS, Singh P (2011) Self emulsifying drug delivery system. J Pharm Res 3: 500-504.

35. Hiral AM, Ami YB, Ramesh BP, Jalpa SP, Tank HM (2013) Self nano emulsifying drug delivery system (SNEDDS): Future aspects. Asian J Pharm Res 3(1): 21-27.

36. Udaya SM, Josephine RLF, Kiran BU (2013) Self nano emulsifying drug delivery systems for oral delivery of hydrophobic drugs. Biomed Pharmacol J 6(2): 355362.

37. Obitte NC, Ofokansi KC, Nzekwe IT, Esimone CO, Okoye IE (2011) Self-nanoemulsifying drug delivery systems based on melon oil and its admixture with a homolipid from Bos indicus for the delivery of indomethacin. Trop J Pharm Res 10(3): 299-307.

38. Thomas N, Holm R, Garmer M, Karlsson JJ, Müllertz A, et al. (2013) Supersaturated self-nanoemulsifying drug delivery systems (Super-SNEDDS) enhance the bioavailability of the poorly water-soluble drug simvastatin in dogs. AAPS Pharm Sci Tech 15(1): 219227.

39. Beg S, Swain S, Singh H.P, Patra CN, Rao MB (2012) Development, optimization, and characterization of solid self-nanoemulsifying drug delivery systems of valsartan using porous carriers. AAPS Pharm Sci Tech 13(4): 1416-1427.

40. Beg S, Jena SS, Patra CN, Rizwan M, Swain S, et al. (2013) Development of solid self-nanoemulsifying granules (SSNEGs) of ondansteron hydrochloride with enhanced bioavailability potential. Colloids Surf B Biointer 101: 414-423.

41. Zhang Q, He N, Zhang L, Zhu F, Chen Q, et al. (2012) The in vitro and in vivo study on self-nanoemulsifying drug delivery system (SNEDDS) based on insulinphospholipid complex. J Biomed Nanotech 8(1): 9097.

42. Elsheikh MA, Elnaggar YSR, Gohar EY, Abdallah OY (2012) Nanoemulsion liquid preconcentrates for raloxifene hydrochloride: Optimization and in vivo appraisal. Int J Nanomed 7: 3787-3802.

43. Lei Y, Qi J, Nie S, Hu F, Pan W, et al. (2012) Solid selfnanoemulsifying cyclosporine a pellets prepared by fluid-bed coating: Stability and bioavailability study. J Biomed Nanotech 8(3): 515-521.

44. Sun M, Han J, Guo X, Li Z, Yang J, Zhang Y, Zhang D (2011) Design, preparation and in vitro evaluation of paclitaxel-loaded self-nanoemulsifying drug delivery system. Asian J Pharm Sci 6(1): 18-25.

45. Jeevana JB, Sreelakshmi K (2011) Design and evaluation of self-nanoemulsifying drug delivery system of flutamide. J Young Pharm 3(1): 4-8.

46. Abhijit AD, Neha D, Rahul D, Mangal N (2010) Self nanoemulsifying drug delivery system: formulation insights, applications and advances. Nanomedicine 5(10): 23-41.

47. Anjan KM, Narasimha MP, Swadeep B, Ranjit PS (2014) Self-emulsifying drug delivery systems (SEDDS): an update from formulation development to therapeutic strategies. Int J PharmTech Res 6(2): 546568.

48. Cole ET, Cade D, Benameur H (2008) Challenges and opportunities in the encapsulation of liquid and semisolid formulations into capsules for oral administration. Adv Drug Deliv Rev 60(6): 747-756.

49. Rodriguez L, Passerini N, Cavallari C, Cini M, Sancin P, et al. (1999) Description and preliminary evaluation of a new ultrasonic atomizer for spray-congealing process. Int J Pharm 183(2): 133-143.

50. Ito Y, Kusawake T, Ishida M, Tawa R (2005) Oral solid gentamicin preparation using emulsifier and adsorbent. J Control Rel 105(1): 23-31.

51. Venkatesan N, Yoshimitsu J, Ito Y, Shibata N, Takada K (2005) Liquid filled nanoparticles as a drug delivery tool for protein therapeutics. Biomaterials 26(34): 7154-7163.

52. Venkatesan N, Yoshimitsu J, Ohashi Y, Ito Y, Sugioka $\mathrm{N}$, et al. (2006) Pharmacokinetic and pharmacodynamic studies following oral administration of erythropoietin mucoadhesive tablets to beagle dogs. Int J Pharm 310(1-2): 46-52.

53. Verreck G, Brewster ME (2004) Melt extrusion-based dosage forms: excipients and processing conditions for pharmaceutical formulations. Bull Tech Gattefossé 24: 85- 95. 


\section{Open Access Journal of Pharmaceutical Research}

54. Breitenbach J (2002) Melt extrusion: from process to drug delivery technology. Eur J Pharm. Biopharm 54(2): 107-117.

55. Rutvij JP, Gunjan JP, Bharadia PD, Pandya VM, Modi DA (2011) Nanoemulsion: Anadvanced concept of dosage form. Int J Pharm Cosmetol 1(5): 122-133.

56. Yashpal S, Tanuj H, Harsh K (2013) Nanoemulsions: A pharmaceutical review. Int J Pharma Prof Res 4(2): 928-935.

57. Charles L, Attama AA (2011) Current state of nanoemulsions in drug delivery. J Biomat Nanobiotech 2: 626-639.

58. Adnan A, Mohammad R, Farhan JA, Zeenat I, Roop KK, et al. (2009) Nanoemulsion components screening and selection: a technical note. AAPS Pharm Sci Tech 10(1): 69-76.

59. Lawrence MJ, Rees GD (2000) Microemulsion-based media as novel drug delivery systems. Adv Drug Deliv Rev 45: 89-121.

60. Kamalinder K Singh, Sharvani K Vingkar (2008) Formulation, antimalarial activity and bio distribution of oral lipid nanoemulsion of primaquine. International Journal of Pharmaceutics 347:136-143.

61. Sheikh Shafiq-un-Nabi, Faiyaz Shakeel, SushmaTalegaonkar, Javed Ali, Sanjula Baboota, et al. (2007) Formulation Development and Optimization Using NanoemulsionTechnique: A Technical Note. AAPS Pharm Sci Tech 8(2).

62. Bhatt P, Madhav S (2011) A detailed review on Nanoemulsion drug delivery system. IJPSR Vol. 2(10): 2482-2489.

63. Farhan Ahmad J, Mushir Ali, Faiyaz Shekel, Cushman Talegaonkar, RoopKhar K (2008) Investigation of Nanoemulsion System for Transdermal Delivery of Domperidone: Ex-vivo andin vivo Studies 4: 381-390.

64. Lawrence MJ, Rees GD (2000) Microemulsion-based media as novel drug deliverysystems. Adv. Drug Deliv Rev 45: 89-121.

65. Narang AS, Delmarre D, Gao D (2007) Stable drug encapsulation in micelles and microemulsions. Int J Pharm 345: 9-25.

66. Constantinides PP (1995) Lipid microemulsions for improving drug dissolution andoral absorption and biopharmaceutical aspects. Pharm Res 12(11): 15611572.

67. Karim A, Gokhale R, Cole M, Sherman J, Yeramian P, et al. (1994) HIV protease inhibitor SC-52151: a novel method of optimizing bioavailability profile via microemulsion drug delivery system. Pharm Res 11: S368.

68. Kawakami K, Yoshikawa T, Hayashi T, Nishihara Y, Masuda K (2002) Microemulsion formulation for enhanced absorption of poorly soluble drugs. II. In vivo study. J Control Release 81(1-2):75-82.

69. Kommuru TR, Gurley B, Khan MA, Reddy IK (2001) Self-emulsifying drug delivery systems (SEDDS) of coenzyme Q10: formulation development and bioavailability assessment. Int J Pharm 212(2): 233246.

70. Kreilgaard M, Pedersen EJ, Jaroszewski JW (2000) NMR characterization and transdermal drug delivery potential of microemulsion systems. J Control Rel 69(3): 421-433.

71. Tenjarla S (1999) Microemulsions: an overview and pharmaceutical applications. Crit Rev Ther Drug Carrier Syst 16(5): 461-521.

72. Attwood D (1994) Microemulsions. In: Kreuer J, Editor. Colloidal drug delivery systems. New York: Marcel Dekker. 31-71.

73. Ravi TPU, Padma T (2011) Nanoemulsions for drug delivery through different routes. Research in Biotechnol 2(3): 1-13.

74. Anton N, Vandamme T (2009) The universality of low-energy nano-emulsification. Int J Pharm 377 (12): 142-147.

75. Mason TG, Graves SM, Wilking JN, Lin MY (2006) Extreme emulsification: formation and structure of nanoemulsions. J Phy Cond Matter 9(1): 193-199.

76. Tadros T, Izquierdo P, Esquena J, Solans C (2004) Formation and stability of Nanoemulsions. Adv. Colloid Interface Sci 108-109: 303-318.

77. Lamaallam S, Bataller H, Dicharry C, Lachaise J (2005) New York: Marcel Dekker Solans C, Esquena J, Forgiarini AM, Uson $\mathrm{N}$, Morales $\mathrm{D}$, Izquierdo $\mathrm{P}$ Nanoemulsions: formation and properties. In: Mittal KL, Shah DO, Editors. Surfactants in solution: 


\section{Open Access Journal of Pharmaceutical Research}

fundamentals and applications. New York: Marcel Dekker Pp: 525.

78. Izquierdo P, Feng J, Esquena J, Tadros TF, Dederen JC, et al. (2005) The influenceof surfactant mixing ratio on nano-emulsion formation by the pit method. J Colloid Interf Sci 285(1): 388-394.

79. Shinoda K, Saito H (1968) The effect of temperature on the phase equilibria and thetypes of dispersions of the ternary system composed of water, cyclohexane, and non-ionic surfactant. J Colloid Interf Sci 26(1): 70-74.

80. Forgiarini A, Esquena J, Gonzalez C, Solans C (2001) Formation of nano-emulsionsby low-energy emulsification methods at constant temperature. Langmuir 17(7): 2076-2083.

81. Pey CM, Maestro A, Solé I, González C, Solans C, Gutiérrez JM (2006) Optimization of nano-emulsions prepared by low-energy emulsification methods at constant temperature using a factorial design study. Colloids Surf A: Physicochem. Eng Aspects 288(1-3): 144-150.

82. Porras M, Solans C, González C, Gutiérrez JM (2008) Properties of water-in-oil (W/O)nano-emulsions prepared by a low-energy emulsification method. Colloids Surf A: Physicochem Eng Aspects 324(1-3): 181-188.

83. Sonneville-Aubrun O, Simonnet JT, L'Alloret F (2004) Nanoemulsions: A new vehiclefor skincare products. Adv Colloids Interf Sci 108-109: 145-149.

84. Solans C, Izquierdo P, Nolla J, Azemar N, Garcia-Celma MJ (2005) Nano-emulsions. Curr Opin Colloid Interf Sci 10 (3-4): 102-110.
85. Wang L, Li X, Zhang G, Dong J, Eastoe J (2007) Oil-inwater nanoemulsions for pesticide formulations. J Colloid Interf Sci 314(1): 230-235.

86. Sole I, Maestro A, Pey CM, Gonzalez C, Solans C, Gutierrez JM (2006) Nanoemulsions preparation by low energy methods in an ionic surfactant system. Colloids Surf A: Physiochem Eng Aspects 288: 138143.

87. Shafiq S, Faiyaz S, Sushma T, Ahmad FJ, Khar RK, et al. (2007) Development and bioavailability assessment of ramipril nanoemulsion formulation. Eur J Pharm Biopharm 66(2): 227-243.

88. Shakeel F, Baboota S, Ahuja A, Ali J, Faisal MS, Shafiq S (2008) Stability evaluation of celecoxib nanoemulsion containing Tween 80. Thai J Pharm Sci 32: 4-9.

89. Shakeel F, Baboota S, Ahuja A, Ali J, Shafiq S (2008) Skin permeation mechanism ofaceclofenac using novel nanoemulsion formulation. Pharmazie 63(8): 580-584.

90. Baboota S, Shakeel F, Ahuja A, Ali J, Shafiq S (2007) Design, development and evaluation of novel nanoemulsion formulations for transdermal potential of celecoxib. Acta Pharm 57(3): 315-332.

91. Mason TG, Wilking JN, Meleson K, Chang CB, Graves SM (2006) Nanoemulsions: formation, structure and physical properties. J Phys Condens Matter 18: 636643.

92. Haritha A, Syed PB, Koteswara RP, Chakravarthi V (2013) A brief introduction to methods of preparation, applications and characterization of nanoemulsion drug delivery systems. Indian J Res Pharm Biotechnol 1 (1): 25-28.

93. Narang AS, Delmarre D, Gao D (2007) Stable drug encapsulation in micelles and microemulsions. Int J Pharm 345(1-2): 9-25. 